\title{
ON THE NEWTONIAN POTENTIAL DUE TO A SURFACE DISTRIBUTION HAVING A DISCONTINUITY OF THE SECOND KIND.
}

\author{
By G. Prasad (Calcutta).
}

Adunanza dell's i giugno 1916.

The object of the following lines is chiefly to point out a case in which the Newtonian potential, due to a surface distribution having a discontinuity of the second kind, is such that the normal derivate does not tend to a limit as the boundary is approached. It is believed that such a case ${ }^{I}$ ) has never been discussed by any of the previous writers on the subject from Prof. Hölder to Prof. Picard. About the end of the paper, I have given a simple case ${ }^{2}$ ) in which the derivate tends to a limit, although the surface distribution has a discontinuity of the second kind.

r. In order to avoid unnecessary complications, I take the surface to be a sphere of unit radius. Let $C$ be the centre of the sphere and $O$ any point on it. Then, taking $O C$ as the $z$-axis and the tangent-plane at $O$ as the $z$-plane, the potential $V$ at any point $(0,0, z)$ on $O C$ may be taken to be $u+U$, where $u$ is the potential due to a small element $S$ of the sphere bounded by the circle $Q O Q^{\prime}$ and $U$ that due to the remaining part of the sphere. It is obvious that $\lim _{z=0} \frac{\partial U}{\partial z}$ exists; also

$$
\frac{\partial u}{\partial z}=-2 z \pi \int_{0}^{a} \frac{\sigma r d r}{\left(z^{2}+r^{2}\right)^{\frac{3}{2}}}
$$

where $\sigma$ is the surface density, $O Q=a$, and the curvature of the element $S$ is neglected, it being taken to be a plane circle with centre $O$ and radius $a$.

2. I proceed to prove that $\lim _{z=0} \frac{\partial u}{\partial z}$ is non-existent provided that $\sigma=\cos \log \frac{\mathrm{I}}{r}$.

I) For the logarithmic potential due to a linear distribution, I have given a similar case in the Bulletin of the Calcutta Mathematical Society, vol. V (1914).

2) For the logarithmic potential due to a linear distribution, Dr. G. Pucciano has given a similar case: G. Pucciano, Studio sui potenziali logaritmici di strato lineare semplice e doppio, e delle loro derivate prime [Rendiconti del Circolo Matematico di Palermo, t. XXIII ( ${ }^{\circ}$ semestre 1907), pp. 374-393]. 
Here, putting $\log \frac{\mathrm{I}}{r}=v$, we have

$$
\begin{aligned}
& -\frac{\mathrm{I}}{2 \chi \pi} \frac{\partial u}{\partial z}=\int_{0}^{a} \frac{r \cos \log \frac{\mathrm{I}}{r} d r}{\left(z^{2}+r^{2}\right)^{\frac{3}{2}}} \\
& =\int_{\log \frac{1}{a}}^{\infty} \frac{e^{-2 v} \cos v d v}{\left(z^{2}+e^{-2 v}\right)^{\frac{3}{2}}} \\
& =\int_{\log \frac{1}{a}}^{\log \frac{1}{z}} \frac{e^{-2 v} \cos v d v}{\left(z^{2}+e^{-2 v}\right)^{\frac{3}{2}}}+\int_{\log \frac{1}{z}}^{\infty} \frac{e^{-2 v} \cos v d v}{\left(z^{2}+e^{-2 v}\right)^{\frac{3}{2}}} \\
& =\int_{\log \frac{1}{a}}^{\log \frac{1}{z}} d v e^{v} \cos v\left\{\mathbf{I}-\frac{3}{2} z^{2} e^{2 v}+\frac{3.5}{2.4} z^{4} e^{4 v}-\cdots \cdots\right\} \\
& +\int_{\log \frac{1}{z}}^{\infty} \frac{d v e^{-2 v} \cos v}{z^{3}}\left\{1-\frac{3}{2} \frac{e^{-2 v}}{z^{2}}+\frac{35}{2 \cdot 4} \frac{e^{-4 v}}{z^{4}}-\cdots \cdots\right\} \\
& =\left[\frac{e^{v}(\sin v+\cos v)}{\mathrm{I}+\mathrm{I}^{2}}-\frac{3}{2} \frac{z^{2} e^{3 v}(\sin v+3 \cos v)}{\mathrm{I}+3^{2}}+\cdots\right]_{\log \frac{\mathrm{I}}{a}}^{\log \frac{\mathrm{I}}{z}} \\
& +\left[\frac{e^{-2 v}(\sin v-2 \cos v)}{z^{3}\left(1+2^{2}\right)}-\frac{3}{2} \frac{e^{-4 v}(\sin v-4 \cos v)}{z^{5}\left(1+4^{2}\right)}+\cdots\right]_{\log \frac{s}{z}}^{\infty} \\
& =\frac{\mathrm{I}}{2} \sin \log \frac{\mathrm{I}}{2}\left\{\left(\frac{\mathrm{I}}{\mathrm{I}+\mathrm{I}^{2}}-\frac{3}{2} \frac{\mathrm{I}}{\mathrm{I}+3^{2}}+\frac{3 \cdot 5}{2 \cdot 4} \frac{\mathrm{I}}{\mathrm{I}+\mathrm{S}^{2}}-\cdots\right)\right. \\
& \left.+\left(\frac{I}{I+2^{2}}-\frac{3}{2} \frac{I}{I+4^{2}}+\frac{3.5}{2.4} \frac{I}{I+6^{2}}-\cdots\right)\right\} \\
& +\frac{I}{z} \cos \log \frac{I}{z}\left\{\left(\frac{I}{I+I^{2}}-\frac{3}{2} \frac{3}{I+3^{2}}+\frac{3.5}{2.4} \frac{5}{I+5^{2}}-\cdots\right)\right. \\
& \left.+\left(\frac{2}{I+2^{2}}-\frac{3}{2} \frac{4}{I+4^{2}}+\frac{3 \cdot 5}{2 \cdot 4} \frac{6}{I+6^{2}}-\cdots\right)\right\}
\end{aligned}
$$$$
-\frac{\mathrm{I}}{a} \frac{\sin \log \frac{\mathrm{I}}{a}+\cos \log \frac{\mathrm{I}}{a}}{\mathrm{I}+\mathrm{I}^{2}}+\text { terms which vanish with } z \text {. }
$$

Now the infinite series in the above expression are all convergent.

Therefore, as $z$ tends to zero, $\frac{\partial u}{\partial z}$ behaves as $A \cos \log \frac{1}{z}+B \sin \log \frac{1}{z}$, where $A$ and $B$ are known constanı. 
Thus it is proved that $\lim _{z=0} \frac{\partial u}{\partial z}$ and, consequently, $\lim _{z=0} \frac{\partial V}{\partial z}$ are non-existent.

3. It is easily seen that, if the distribution be

$$
\sigma=\sum_{i}^{\infty} \frac{f\left(\theta-\omega_{n}\right)}{n^{2}}
$$

where $\theta$ is the angle made by any radius $C P$ with a fixed diameter, $\left\{\omega_{n}\right\}$ is the aggregate of rational numbers between $o$ and $\pi$, and $f(x)$ is $\cos \log \frac{\mathrm{I}}{x}$ or $\cos \log \frac{\mathrm{I}}{-x}$ according as $x$ is positive or negative, the normal derivate at any point $Q$ on the radius $C P$ makes infinite number of fluctuations (without tending to a limit) as $Q$ approaches $P$, provided that $\theta$ be a member of the aggregate.

4. I proceed now to prove that $\lim _{z=0} \frac{\partial u}{\partial z}$ and, consequently, $\lim _{z=0} \frac{\partial V}{\partial z}$ are existent provided that $\sigma=\cos \frac{\mathrm{I}}{r}$.

Putting $\frac{\mathrm{I}}{r}=v$, we have

$$
-\frac{\mathrm{I}}{2 z \pi} \frac{\partial u}{\partial z}=\int_{0}^{a} \frac{r \cos \frac{\mathrm{I}}{r} d r}{\left(z^{2}+r^{2}\right)^{\frac{3}{2}}}=\int_{\frac{1}{a}}^{\infty} \frac{\cos v d v}{\left(\mathrm{I}+v^{2} z^{2}\right)^{\frac{3}{2}}} .
$$

Now $\frac{1}{\left(1+v^{2} z^{2}\right)^{\frac{3}{2}}}$ is always positive and its greatest value is $\frac{1}{\left(1+\frac{z^{2}}{a^{2}}\right)^{\frac{3}{2}}}$ Therefore

$$
\left|\int_{\frac{1}{a}}^{\infty} \frac{\cos v d v}{\left(\mathrm{I}+v^{2} z^{2}\right)^{\frac{3}{2}}}\right|<\frac{\mathrm{I}}{\left(\mathrm{I}+\frac{z^{2}}{a^{2}}\right)^{\frac{3}{2}}}
$$

Hence $\frac{\partial u}{\partial z}$ is numerically less than $2 \pi z$. Therefore $\lim _{z=0} \frac{\partial u}{\partial z}=0$.

Calcutta, 1o May 1916.

G. PRASAD. 